\title{
Suppression of proliferation, migration and invasion in non-small cell lung cancer cells via profilin 2 inhibition by microRNA-194
}

\author{
Jinjiao Zhang ${ }^{1}$, Yong Gao ${ }^{2 \star}$, Lei Zhang ${ }^{1}$, Tao Wang ${ }^{1}$, Hong $\mathrm{Xu}^{1}$, Gang Chen ${ }^{1}$ \\ ${ }^{1}$ Department of Thoracic Surgery, ${ }^{2}$ Department of Emergency, The Fifth Affiliated Hospital of Southern Medical University, \\ Guangzhou 510900, China
}

*For correspondence: Email: yonggao_smu@163.com; Tel: +86-20-61780161

Sent for review: 10 February 2018

Revised accepted: 25 April 2018

\begin{abstract}
Purpose: To investigate the anti-tumor effect of microRNA-194 (miR-194) and possible relationship with profilin 2.

Methods: The expressions of miR-194 and profilin 2 were investigated via bioinformatic analysis and were further verified using quantitative real-time polymerase chain reaction (qRT-PCR) in both normal and non-small cell lung cancer (NSCLC) cells. Dual luciferase reporter assay, together with transfection, was applied to determine the effect of miR-194 and profilin 2 on proliferation, migration and invasion, and its underlying mechanisms.

Results: Relatively low expression of miR-194 and high expression of profilin 2 in cancerous lung tissue were found through bioinformatic analysis and further confirmed by qRT-PCR on normal and NSCLC cell lines. Profilin 2 was the direct target of miR-194. Also, miR-194 overexpression inhibited the proliferation, migration and invasion of NSCLC cells, and this suppressive effect was partially reversed by transfection of pcDNA3.1-PFN2 plasmid.

Conclusion: The results show that miR-194 plays a tumor suppressor role in NSCLC development. It inhibits the proliferation, migration and invasion of NSCLC cells via profilin 2 suppression. Interventions targeting the miR-194/profilin 2 axis may therefore serve as a clinical strategy for the prevention and treatment of NSCLC metastases.
\end{abstract}

Keywords: Non-small cell lung cancer, MicroRNA-194, Profilin 2, Proliferation, Migration, Invasion

\begin{abstract}
This is an Open Access article that uses a funding model which does not charge readers or their institutions for access and distributed under the terms of the Creative Commons Attribution License (http://creativecommons.org/licenses/by/4.0) and the Budapest Open Access Initiative (http://www.budapestopenaccessinitiative.org/read), which permit unrestricted use, distribution, and reproduction in any medium, provided the original work is properly credited.
\end{abstract}

Tropical Journal of Pharmaceutical Research is indexed by Science Citation Index (SciSearch), Scopus, International Pharmaceutical Abstract, Chemical Abstracts, Embase, Index Copernicus, EBSCO, African Index Medicus, JournalSeek, Journal Citation Reports/Science Edition, Directory of Open Access Journals (DOAJ), African Journal Online, Bioline International, Open-J-Gate and Pharmacy Abstracts

\section{INTRODUCTION}

Lung cancer is a major cause of cancerassociated mortality worldwide. Among various subtypes, non-small cell lung cancer (NSCLC) is one of the most common which accounts for 80 $\%$ of all diagnosed lung cancer cases $[1,2]$. Compared to small cell lung cancer (SCLC), patients with NSCLC are less sensitive to radiochemotherapy, have higher recurrence rate, and thus lower long-term survival rate [3]. Excessive proliferation and metastasis of cancer cells are major reasons for poor prognosis of NSCLC patients [4]. Therefore, identifying related genes and possible underlying mechanisms is of great 
importance in improving early diagnosis of NSCLC and targeted therapy development.

microRNA, a short non-coding RNA with length between 18 and 25 amino acids, is generally assumed to play a negative role in gene regulation in eukaryotic cells. Bounded to the 3'untranslated region (UTR), microRNA can inhibit target gene transcription, even leading to the degradation of targeted mRNA. Tumor development is often accompanied with abnormal microRNA expression and distribution. Thus, microRNA is not only a possible biomarker for early diagnosis, but also a target for future targeted therapy with great potential [5]. Relatively low expression of microRNA-194 (miR-194) in cancers such as hepatocellular carcinoma, pancreatic cancer, melanoma and ovarian cancer have been widely reported previously. Abnormal miR-194 expression might be related to tumor progression, and thus suggesting poor prognosis [6,7]. Zhang et al reported that overexpression of miR-194 can inhibit migration and invasion in glioma cells [8]. Moreover, the proliferation of melanoma cells was suppressed by miR-194 overexpression [9]. However, the specific role of miR-194 in NSCLC and further underlying mechanisms have yet not been unveiled.

Profilin, with a molecular weight between 12 and $15 \mathrm{kDa}$, is a family of actin-binding protein constitutively expressed in eukaryotic cells, including profilin 1, 2, 3 and 4 . These proteins are involved in cell proliferation, differentiation, and cell signal transduction by regulating polymerization and depolymerization of actin $[10,11]$. Profilin family, especially profilin 2 , has been thoroughly studied in the field of tumorigenesis and development, however, with inconsistent results. Kim et al reported that profilin 2 promotes migration and invasion of human colorectal cancer cell line HT29 [10], while suppressing cell proliferation in oral squamous cell carcinoma [12]. Moreover, preliminary work shown done that profilin 2 is relatively overexpressed in NSCLC 95D cells and is possibly related to epithelial-mesenchymal transition (EMT) [13].

Therefore, the present study was to investigate the anti-tumor effect of miR-194 and possible relationship with profilin 2 in NSCLC.

\section{EXPERIMENTAL}

\section{Bioinformatic analysis}

The potential target gene of miR-194 was predicted using miRanda/mirSVR tool. In order to obtain the microRNA expression profile changes and miR-194 level changes of patients with NSCLC, we retrieved a high-throughput sequencing data from patients with lung squamous cell carcinoma (SCLC) in The Cancer Genome Atlas (TCGA) database. Selected dataset include 45 cases of healthy control tissues and 332 tumor tissues. In the Gene Expression Omnibus (GEO) database, we retrieved a microRNA microarray data about lung adenocarcinoma (accession ID: GSE51853) and different expression gene analysis was conducted for getting the microRNA expression profile. These dataset include 126 normal tissues and 76 tumor tissues. Moreover, for estimating the expression change of profilin in patients with NSCLC, two relevant cohort study data (Landi Cohort and Talbot Cohort) were collected from Oncomine database.

\section{Cell culture}

Human NSCLC cell lines (SK-MES-1, H1299, A549, HCC827 and H460) and human normal lung cell line (MRC-5) were purchased from the Cell Bank of the Chinese Academy of Science (Shanghai, China). MRC-5 and A549 cells were cultured in DMEM, while SK-MES-1, H1299, $\mathrm{H} 460$ and HCC827 cells were culture in RPMI1640 medium (with $10 \%$ fetal bovine serum), supplemented with $100 \mathrm{U} / \mathrm{mL}$ penicillin and 100 $\mu \mathrm{g} / \mathrm{mL}$ streptomycin (all from Gibco, Carlsbad, CA, USA). Cells were cultured in a humidified environment with $5 \% \mathrm{CO}_{2}$ at $37^{\circ} \mathrm{C}$.

\section{Quantitative real-time PCR (qRT-PCR)}

Total RNA was extracted using TRIzol (Gibco) from A549 cells. miR-194 was reversed transcript using miScript II RT kit (Qiagen, Dusseldorf, Germany) and SNORD-48 was set as internal reference. For profilin 2 mRNA, SuperScript Master kit (Thermo Fisher Scientific) were used with GAPDH as internal reference. The fold change of targeted genes expressions were determined by $2^{-\Delta \Delta \mathrm{Ct}}$ method.

\section{Western blotting}

Western blot was conducted as previously described [14] using primary antibodies specific for profilin 2 and GAPDH (Sigma-Aldrich, St. Louis, MO, USA). Image J (US NIH, Bethesda, $M D$, USA) was used to analyze the optical density of each protein band.

\section{Cell transfection}

Cell transfection was performed using Lipofectamine RNAiMAX kit (Thermo Fisher 
Scientific, Waltham, MA, USA) for miR-194 mimic and siRNA, and Lipofectamine 2000 kit (Thermo Fisher Scientific) for pcDNA3.1-PFN2 plasmid, respectively. All the procedures were performed strictly according to the manufacturer's instruction.

\section{Dual luciferase reporter assay}

The 3'-UTR sequence of profilin 2 mRNA containing wild-type or mutant miR-194 binding sites was synthesized and cloned into the PsiCHECK2 fluorescent reporter plasmid (Promega, Madison, WI, USA), respectively. Transfected cells $\left(1 \times 10^{4}\right.$ cells/well) were seeded in 96-well plates for $48 \mathrm{~h}$. Relative luciferase activity was determined by Dual-Luciferase Reporter Assay System (Promega).

\section{Cell viability assay}

Transfected A549 cells $\left(3 \times 10^{3}\right.$ cells/well $)$ seeded in 96-well plates were further cultured for 24, 48 and $72 \mathrm{~h}$, respectively. Cell viability was determined using CCK-8 kit (Jiancheng, Nanjing, China) according to the manufacturer's instruction. The absorbance at $450 \mathrm{~nm}$ was determined with Multiskan (Thermo Fisher Scientific).

\section{Scratch assay}

A549 cells $\left(4 \times 10^{5}\right.$ cells/well) were seeded in a 6 -well plate to form a monolayer. The scratch assay was performed using a p200 pipet tip [15]. Photos were taken $72 \mathrm{~h}$ after the first scratch, and the area of uncover zone were recorded.

\section{Transwell assay}

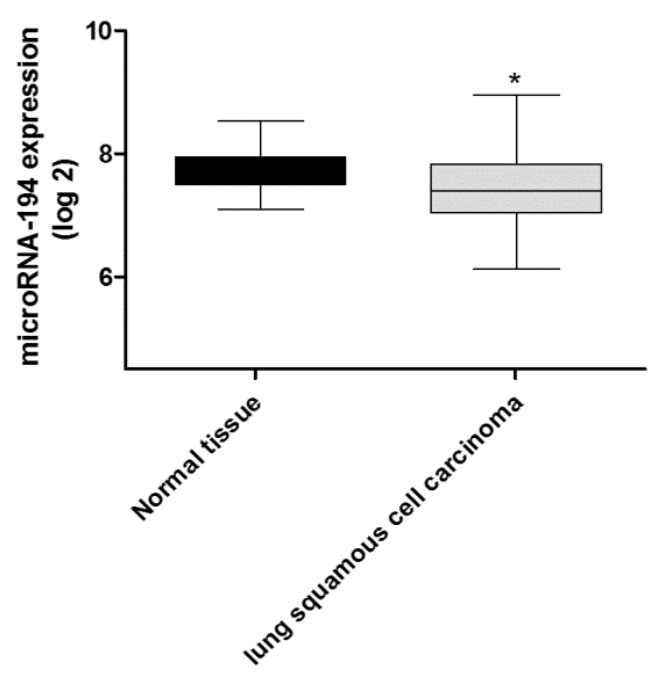

A549 cells $\left(1 \times 10^{5}\right.$ cells/well $)$ were inoculated into the upper chamber of Matrigel-coated Transwell ( $8 \mu \mathrm{m}$ pore size). After $16 \mathrm{~h}$ incubation, migrated cells on were fixed with $4 \%$ formalin, and further stained with Giemsa and counted under the microscope.

\section{Statistical analysis}

All data are presented as mean $\pm S D(n=3)$. Statistical analysis using Student's $t$ test, MannWhitney $U$ test or one-way ANOVA followed by Dunnett's test was performed. $P$-value was calculated using Prism software (GraphPad, La Jolla, CA, USA), and values $<0.05$ were considered statistically significant.

\section{RESULTS}

Bioinformatic data on miR-194 and profilin expressions in patients with NSCLC

According to the dataset retrieved from TCGA, the median level of miR-194 was lower in SCLC tissues compared with matched normal tissues (Figure 1, fold change $=0.8, p<0.05$ ). The microarray data from GEO also showed that miR-194 level decreased in lung adenocarcinoma tissues compared with normal tissue (Figure 1, $p<0.05$ ). The data from Oncomine database demonstrated higher profilin 2 level both in SCLC tissues and lung adenocarcinoma tissues (Figure 2). These results indicated that miR-194 and profilin may play a role in the development of NSCLC.

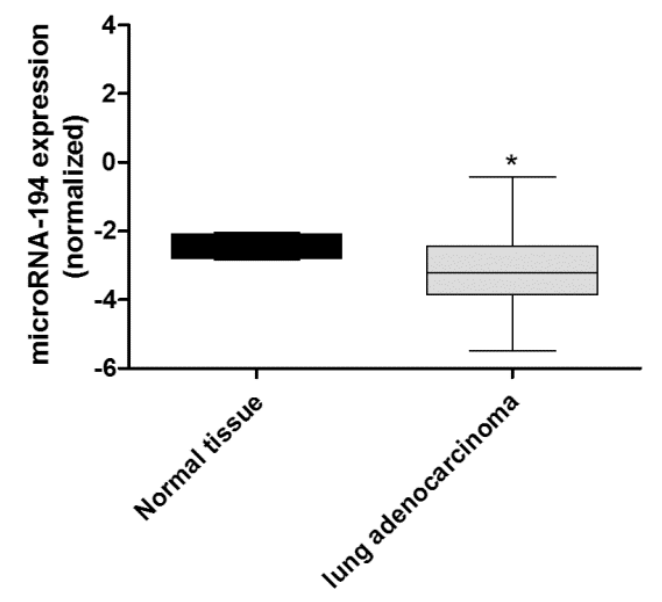

Figure 1: MiR-194 expression in normal and cancerous lung tissue. Datasets were retrieved from TCGA and GEO; *: $p<0.05$ vs normal tissue 


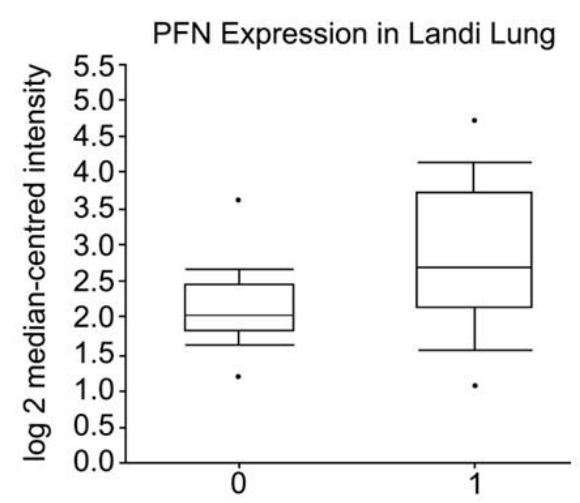

\section{Legend}

$0:$ No value (49)

1: Lung adenocarcinoma (58)

Landi Lung

PloS One $2008 / 02 / 20 \quad 107$ samples
mRNA 12,624 measured genes
Human Genome U133A array

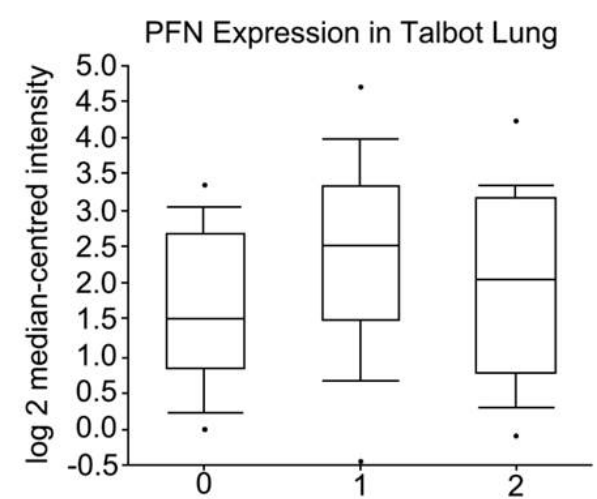

\section{Legend}

$0:$ No value (28)

1 : Squamous cell lung carcinoma (34)

2: Tongue Squamous cell carcinoma (31)

Talbot Lung

Cancer Res $2005 / 04 / 15 \quad 93$ samples
mRNA 8,603 measured genes
Human Genome U95A-Av2 array

Figure 2: Profilin 2 expression in normal and cancerous lung tissue; Dataset was retrieved from Oncomine database
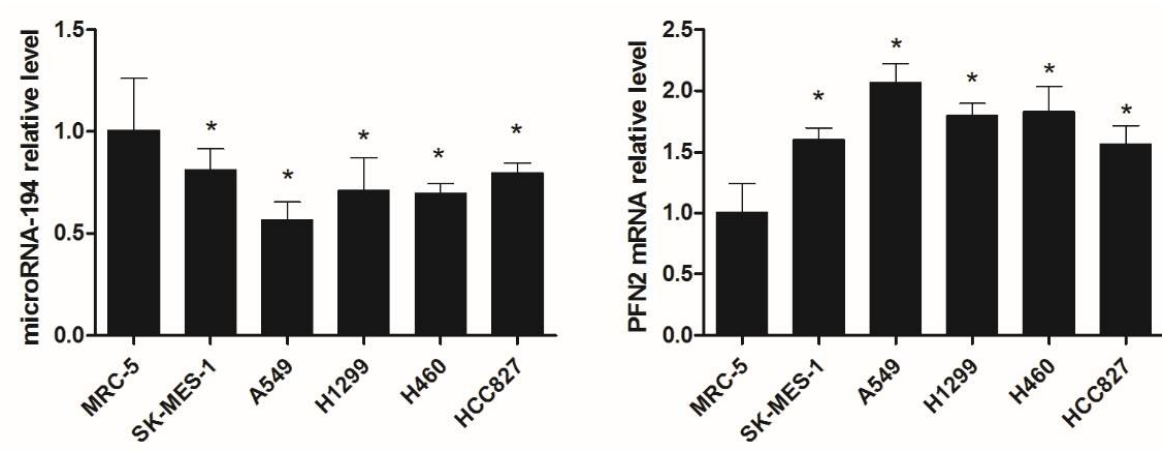

Figure 3: Relative expression level of miR-194 and profilin 2 in normal (MRC-5) and cancerous (SK-MES-1, A549, H1299, H460 and HCC827) lung cell lines; ${ }^{*} p<0.05$ vs MRC-5

\section{MiR-194 and profilin 2 expression in different cell lines}

As shown in Figure 3, the relative level of miR194 in NSCLC cell lines was lower than that in normal human lung cell line MRC- 5 cells $(p<$ 0.05 ), while the level of profilin 2 mRNA was higher than that in MRC- 5 cells $(p<0.05)$. Those cell lines with lower miR-194 levels had higher profilin 2 mRNA levels.

\section{Profilin 2, a downstream target of miR-194}

Results predicted by TargetScan and miRanda/mirSVR software suggested that profilin 2 is a potential target of miR-194. Dual luciferase reporter assay revealed that the fluorescence intensity of cells transfected with PsiCHECK2PFN2-WT+miR-194 mimic was significantly reduced compared to cells transfected with PsiCHECK2-PFN2-WT+mimic negative control (Figure $4, p<0.05$ ). However, the fluorescence intensity of cells transfected with PsiCHECK2PFN2-MUT+miR-194 mimic was comparable to those transfected with PsiCHECK2-PFN2MUT+mimic negative control $(p>0.05)$, suggesting that the 3'-UTR region of profilin 2 mRNA can be the direct binding site of miR-194. Results from qRT-PCR (Figure 5) and Western blot (Figure 6) showed that both profilin 2 mRNA and protein levels were decreased in miR-194 mimic transfected cells $(p<0.05)$. 

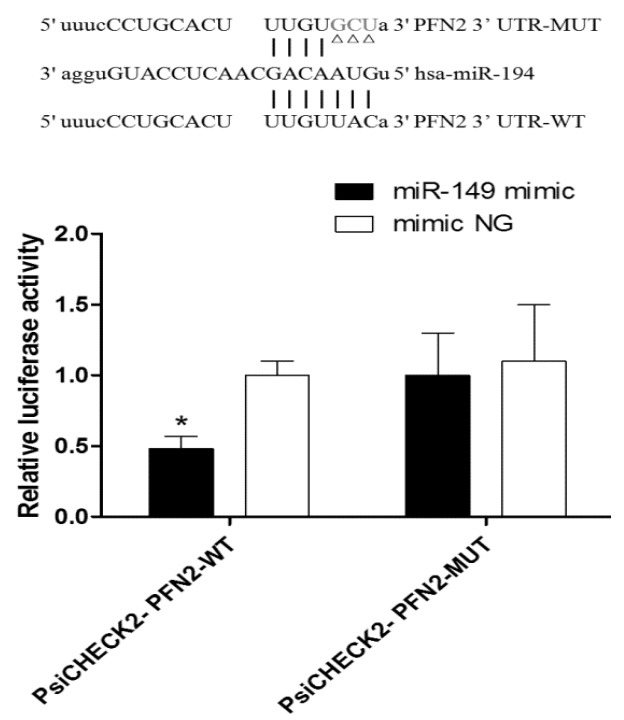

Figure 4: Dual luciferase reporter assay. A549 cells were transfected as indicated. WT: wild-type; MUT: mutant; NG: negative; ${ }^{*} p<0.05$ vs mimic negative control

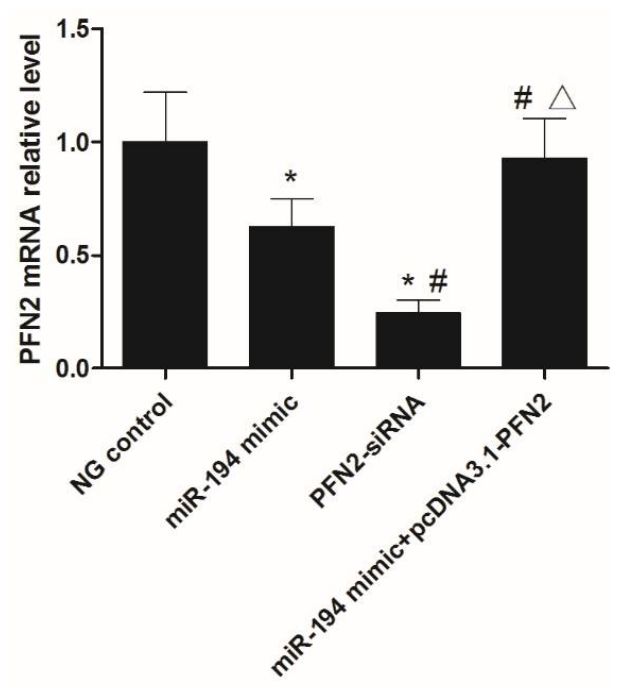

Figure 5: Profilin 2 mRNA level determined by qRTPCR. A549 cells were transfected as indicated. NG: negative; ${ }^{*} p<0.05$ vs control; $\# p<0.05$ vs miR-194 mimic; $\Delta p<0.05$ vs PFN2-siRNA

\section{Effect of miR-194/profilin 2 axis on A549 cell proliferation, migration and invasion}

The CCK-8 cell viability assay showed that the relative proliferation rate of A549 cells overexpressed with miR-194 was lower than that of negative control (Figure $7, p<0.05$ ). The in vitro scratch assay revealed that, the migration of cells was slower after miR-194 overexpression, and the gap length at $72 \mathrm{~h}$ was larger than that of the negative control group (Figure 8, $p<0.05$ ). Transwell assay showed that the number of migrated cells was decreased after miR-194 overexpression, compared with the negative control group (Figure 9, $p<0.05$ ). Similar to the effect of miR-194 overexpression, cell proliferation was inhibited and the migration and invasion abilities also decreased after silencing profilin 2 using siRNA. However, the transfection of miR-194 mimic into pcDNA3.1-PFN2transfected A549 cells partially restored the proliferation, migration and invasion ability, compared with the cells overexpressing miR-194 only, which indicated that the recovery of profilin 2 levels partially abolished the tumor suppressor effect of miR-194. The above results suggest that miR-194 may play a suppressor effect on NSCLC cells through targeted inhibition of profilin 2.
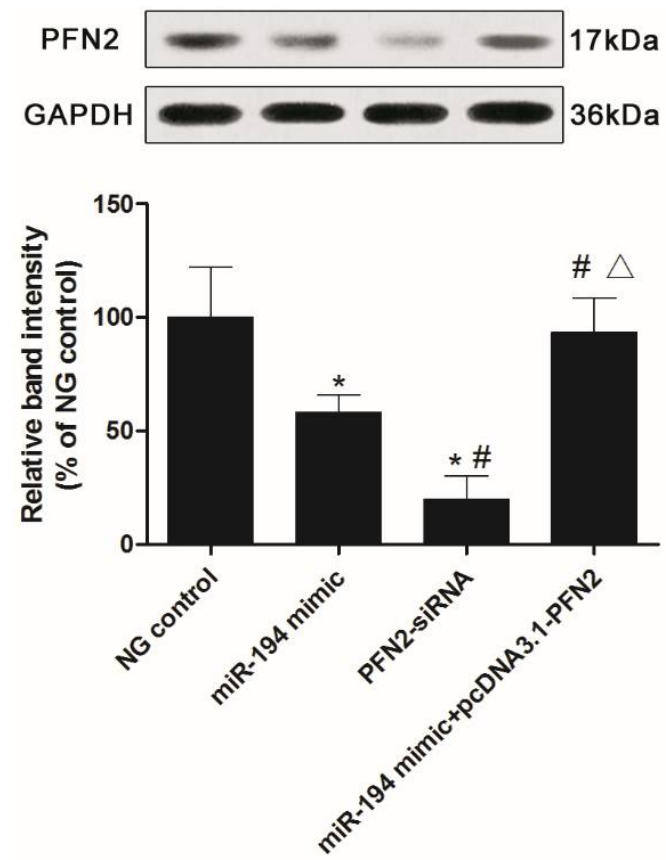

Figure 6: Profilin 2 protein level determined by Western blot. A549 cells were transfected as indicated. NG: negative; ${ }^{*} p<0.05$ vs control; ${ }^{\#} p<0.05$ vs miR-194 mimic; $\Delta p<0.05$ vs PFN2-siRNA

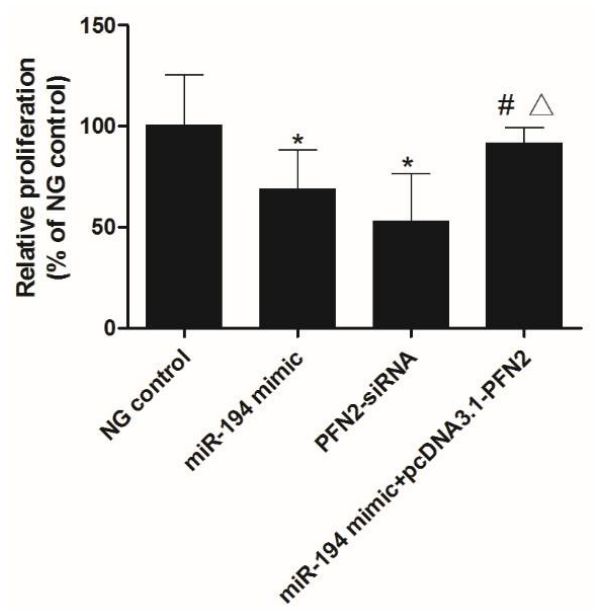

Figure 7: Relative proliferation rate determined by CCK-8 assay. A549 cells were transfected as indicated. NG: negative; ${ }^{*} p<0.05$ vs control; ${ }^{\#} p<0.05$ vs miR-194 mimic; $\Delta p<0.05$ vs PFN2-siRNA 

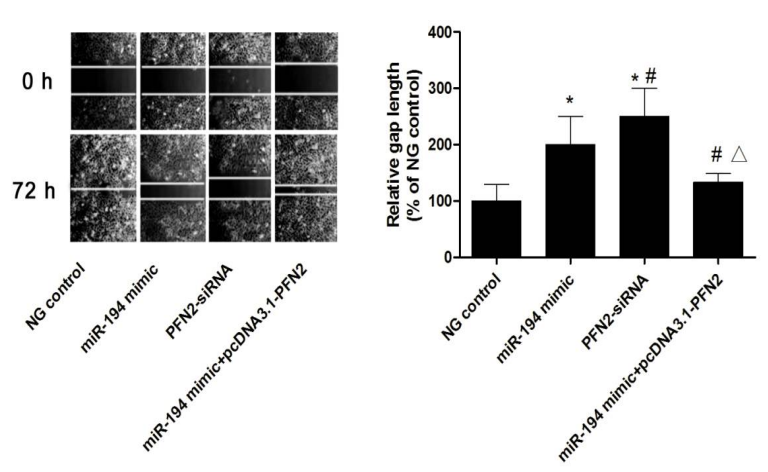

Figure 8: Cell migration ability determined by in vitro scratch assay. A549 cells were transfected as indicated. NG: negative; * $p<0.05$ vs control; ${ }^{*} p<0.05$ vs miR-194 mimic; $\Delta p<0.05$ vs PFN2-siRNA
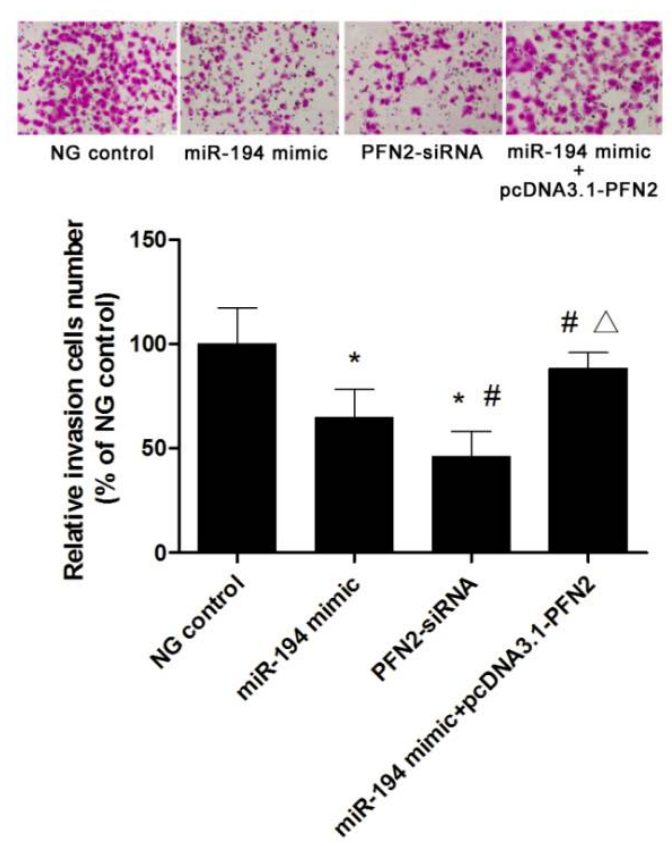

Figure 9: Cell invasion ability determined by Transwell assay. A549 cells were transfected as indicated. NG: negative; ${ }^{*} p<0.05$ vs control; ${ }^{\#} p<0.05$ vs miR-194 mimic; $\Delta p<0.05$ vs PFN2-siRNA

\section{DISCUSSION}

In our present study, results from bioinformatic analysis showed that there were abnormal expressions of miR-194 and profilin 2 in normal and cancerous lung tissues, with relatively low miR-194 expression and high profilin 2 expression in NSCLC patients. The results were further confirmed by in vitro PCR assay using different NSCLC cell lines, compared to normal human lung cells, which suggest possible relationship between these two molecules. Further dual luciferase reporter assay showed that profilin 2 is the direct target of miR-194 in A549 cells. Overexpression of miR-194 could suppress profilin 2 expression both at mRNA and protein level, and further inhibit proliferation, migration and invasion of A549 cells. Similar results were found in profilin 2-knockdown A549 cells using siRNA interference. Of note, the suppressive effect was partially reversed by overexpression of profilin 2 .

Previous studies related to miR-194 mainly focused on its suppressive effect on cell migration and invasion via EMT-related genes and signaling pathways. Meng et al reported that miR-194 is relatively lowly expressed in mesenchymal-like hepatic epithelial cell [7]. Overexpression of miR-194 can down-regulated mesenchymal cell marker expression, indicating the EMT process was inhibited. The metastasis of liver cancer cells was also suppressed in an animal model [7]. Similar expression pattern of miR-194 was also found in gastric cancer cells. miR-194 transfection could reverse the EMT progression, and thus inhibit migration and invasion of gastric cancer cell [16]. BMI-1, an important EMT inducer, is also a potential target of miR-194 in endometrial cancer cells [17]. Moreover, Kong et al reported that miR-194 could inhibit NudC nuclear distribution protein (hNUDC) expression, and thus suppresses migration and invasion of both PC-3 and DU-145 human prostate cancer cell lines [18]. Besides, miR-194 also exerted its anti-proliferative effect on osteosarcoma cells via CDH2 and IGF1R [19]. Furthermore, miR-194 exhibited proapoptotic effect on melanoma cells by modulating PI3K/AKT/FoxO3a and p53/p21 signaling pathway [9].

Currently, there are few studies on the role and underlying mechanism of miR-194 in NSCLC. Zhou et al found that there is relatively low expression of miR-194 in NSCLC patients [20]. After the restoration of miR-194 level in vitro, the proliferation of tumor cells was inhibited, suggesting that the anti-tumor effect of miR-194 may be through the inhibition of its target gene hNUDC [20]. However, due to small sample size, multiple downstream targets and complex regulation network of microRNAs, more studies were warranted to validate the possible target genes of miR-194 and reveal its role in the development of NSCLC.

In the present study, by using bioinformatic analysis, we analyzed large data from TCGA database. The results suggest that there is low expression of miR-194 in NSCLC patients, which is consistent with the previous results. Moreover, the bioinformatics results were confirmed by in vitro assays. After transfection with the miR-194 mimic sequence, we found that the proliferation, migration and invasion of A549 cells were 
inhibited, suggesting the tumor suppressor function of miR-194 in NSCLC.

In order to further reveal the mechanism of action of miR-194, we analyzed the potential target gene profilin 2 in NSCLC. Bioinformatic analysis of two related cohort findings found that patients with NSCLC had high expression of profilin 2. In vitro analysis of NSCLC cell lines revealed that not only the profilin 2 level in the NSCLC cell lines was higher than in the normal lung cancer cell line, but the profilin 2 in the NSCLC cell line with lower miR-194 was rather higher. Further dual luciferase reporter assays, qRT-PCR and Western blot assays also validated profilin 2 as the possible target of miR-194.

At present, there are only few studies on the specific role of profilin 2 in NSCLC, while research have found that profilin 2 can promote cell migration and invasion in other types of cancer. Kim et al. found that reducing the expression of profilin 2 in colorectal cancer stem cells HT29 can not only inhibit the EMT process, but also inhibit cell migration and invasion [10]. Cui et al found that profilin 2 levels correlate with the clinicopathological features of esophageal cancer, with a poorer prognosis for patients with higher profilin 2 levels [21].

Downregulation of profilin 2 in vitro can inhibit esophageal cancer cell proliferation, migration and invasion, accompanied by changes in EMT markers, with significant decrease of mesenchymal markers [21]. These biological effects of profilin 2 may be achieved through the regulation of some signaling pathways, such as PI3K/AKT and TGF- $\beta /$ Smad [22]. This present study found that cell proliferation, migration and invasion of A549 were inhibited after the silencing of profilin 2. Overexpression of profilin 2 can eliminate the tumor suppressor effect of miR194 overexpression, manifested as promoted cell proliferation, migration and invasion. Furthermore, the investigation of the effect of miR-194/profilin 2 axis on EMT of NSCLC cells is necessary in future studies.

\section{CONCLUSION}

The findings of this study suggest that dysregulated miR-194/profilin 2 axis is involved in the development of NSCLC. MiR-194 plays a tumor suppressor role in NSCLC development. It inhibits the proliferation, migration and invasion of NSCLC cells by targeting profilin 2 . Interventions targeting the miR-194/profilin 2 axis may therefore serve as a clinical strategy for the prevention and treatment of NSCLC metastases.

\section{DECLARATIONS}

\section{Acknowledgement}

The work is supported by Medical Scientific Research Foundation of Guangdong Province, China (B2018273).

\section{Conflict of Interest}

No conflict of interest associated with this work.

\section{Contribution of Authors}

We declare that this work was done by the authors named in this article and all liabilities pertaining to claims relating to the content of this article will be borne by the authors. Jinjiao Zhang and Yong Gao conceived and designed the study; Jinjiao Zhang, Gang Chen, Hong Xu and Lei Zhang collected and analyzed the data; Jinjiao Zhang and Tao Wang wrote the manuscript. All authors have read the manuscript and approved for publication.

\section{REFERENCES}

1. Zhan B, Lu D, Luo $P$, Wang B. Prognostic Value of Expression of MicroRNAs in Non-Small Cell Lung Cancer: A Systematic Review and Meta-Analysis. Clin Lab 2016; 62: 2203-2211.

2. Shi YK, Zang QL, Li GX, Huang Y, Wang SZ. Increased expression of microRNA-301a in nonsmall-cell lung cancer and its clinical significance. $J$ Cancer Res Ther 2016; 12: 693-698.

3. Wang W, Chen J, Dai J, Zhang B, Wang F, Sun $Y$. MicroRNA-16-1 Inhibits Tumor Cell Proliferation and Induces Apoptosis in A549 Non-Small Cell Lung Carcinoma Cells. Oncol Res 2016; 24: 345-351.

4. Zhang J, Fu J, Pan $Y$, Zhang $X$, Shen L. Silencing of miR-1247 by DNA methylation promoted non-small-cell lung cancer cell invasion and migration by effects of STMN1. Onco Targets Ther 2016; 9: 7297-7307.

5. Hou J, Meng F, Chan LW, Cho WC, Wong SC. Circulating Plasma MicroRNAs As Diagnostic Markers for NSCLC. Front Genet 2016; 7: 193.

6. Krutzfeldt J, Rosch N, Hausser J, Manoharan M, Zavolan M. Stoffel M. MicroRNA-194 is a target of transcription factor 1 (Tcf1, HNF1alpha) in adult liver and controls expression of frizzled-6. Hepatol 2012; 55: 98-107.

7. Meng Z, Fu X, Chen X, Zeng S, Tian Y, Jove R, Xu R, Huang W. miR-194 is a marker of hepatic epithelial cells and suppresses metastasis of liver cancer cells in mice. Hepatol 2010; 52: 2148-2157.

8. Zhang X, Wei C, Li J, Liu J, Qu J. MicroRNA-194 represses glioma cell epithelialtomesenchymal transition by targeting Bmi1. Oncol Rep 2017; 37: 1593-1600. 
9. Bai $M$, Zhang $M$, Long $F$, $Y u N$, Zeng $A$, Zhao $R$. Circulating microRNA-194 regulates human melanoma cells via PI3K/AKT/FoxO3a and p53/p21 signaling pathway. Oncol Rep 2017; 37: 2702-2710.

10. Kim MJ, Lee YS, Han GY, Lee HN, Ahn C, Kim CW. Profilin 2 promotes migration, invasion, and stemness of HT29 human colorectal cancer stem cells. Biosci Biotechnol Biochem 2015; 79: 1438-1446.

11. Zaidi AH, Raviprakash N, Mokhamatam RB, Gupta P, Manna SK. Profilin potentiates chemotherapeutic agents mediated cell death via suppression of NF-kappaB and upregulation of p53. Apoptosis 2016; 21: 502-513.

12. Ma $C Y$, Zhang $C P$, Zhong LP, Pan HY, Chen WT, Wang LZ, Andrew OW, Ji T, Han W. Decreased expression of profilin 2 in oral squamous cell carcinoma and its clinicopathological implications. Oncol Rep 2011; 26 : 813-823.

13. Yan J, Ma C, Gao Y. MicroRNA-30a-5p suppresses epithelial-mesenchymal transition by targeting profilin-2 in high invasive non-small cell lung cancer cell lines. Oncol Rep 2017; 37: 3146-3154.

14. Bian D, Zhang J, Wu X, Dou Y, Yang Y, Tan Q, Xia Y, Gong Z, Dai Y. Asiatic acid isolated from Centella asiatica inhibits TGF-beta1-induced collagen expression in human keloid fibroblasts via PPAR-gamma activation. Int J Biol Sci 2013; 9: 1032-1042.

15. Liang CC, Park AY, Guan JL. In vitro scratch assay: a convenient and inexpensive method for analysis of cell migration in vitro. Nat Protoc 2007; 2: 329-333.
16. Li Z, Ying $X$, Chen $H$, Ye $P$, Shen $Y$, Pan W, Zhang $L$. MicroRNA-194 inhibits the epithelial-mesenchymal transition in gastric cancer cells by targeting FoxM1. Dig Dis Sci 2014; 59: 2145-2152.

17. Dong P, Kaneuchi M, Watari H, Hamada J, Sudo S, Ju J, Sakuragi N. MicroRNA-194 inhibits epithelial to mesenchymal transition of endometrial cancer cells by targeting oncogene BMI-1. Mol Cancer 2011; 10:99.

18. Kong Q, Chen XS, Tian T, Xia XY, Xu P. MicroRNA-194 suppresses prostate cancer migration and invasion by downregulating human nuclear distribution protein. Oncol Rep 2017; 37: 803-812.

19. Han K, Zhao T, Chen X, Bian N, Yang T, Ma Q, Cai C, Fan $Q$, Zhou $Y$, Ma B. microRNA-194 suppresses osteosarcoma cell proliferation and metastasis in vitro and in vivo by targeting $\mathrm{CDH} 2$ and IGF1R. Int $\mathrm{J}$ Oncol 2014; 45: 1437-1449.

20. Zhou L, Di Q, Sun B, Wang X, Li M, Shi J. MicroRNA-194 restrains the cell progression of non-small cell lung cancer by targeting human nuclear distribution protein C. Oncol Rep 2016; 35: 3435-3444.

21. Cui XB, Zhang SM, Xu YX, Dang HW, Liu CX, Wang LH, Yang L, Hu JM, Liang WH, Jiang JF, et al. PFN2, a novel marker of unfavorable prognosis, is a potential therapeutic target involved in esophageal squamous cell carcinoma. J TransI Med 2016; 14: 137.

22. Tang YN, Ding WQ, Guo XJ, Yuan XW, Wang DM, Song JG. Epigenetic regulation of Smad2 and Smad3 by profilin-2 promotes lung cancer growth and metastasis. Nat Commun 2015; 6: 8230. 